Annuaire suisse de politique de développement

$17 \mid 1998$

Propriété intellectuelle : quels enjeux pour les pays en développement ?

\title{
5. Développement humain
}

\section{(2) OpenEdition}

\section{Journals}

Édition électronique

URL : http://journals.openedition.org/aspd/760

DOI : 10.4000/aspd.760

ISSN : 1663-9669

Éditeur

Institut de hautes études internationales et du développement

Édition imprimée

Date de publication : 1 avril 1998

Pagination : 215-238

ISSN : 1660-5934

Référence électronique

«5. Développement humain », Annuaire suisse de politique de développement [En ligne], 17 | 1998, mis en ligne le 31 juillet 2012, consulté le 08 septembre 2020. URL : http://journals.openedition.org/aspd/ 760 ; DOI : https://doi.org/10.4000/aspd.760 


\section{DÉVELOPPEMENT HUMAIN}

\subsection{ORGANISATION DES NATIONS UNIES}

1997 aura été une année importante pour l'Organisation des Nations Unies puisqu'un nouveau secrétaire général, Kofi Annan, en a pris la direction depuis le mois de janvier. Dans les six mois qui suivirent sa nomination le nouveau secrétaire présentait un train de mesures visant la réforme de l'institution. Bien que non-membre, la Suisse contribue de manière significative au système des Nations Unies. Cependant, suite à une motion d'un parlementaire, le dossier visant une adhésion formelle à l'ONU a été rouvert. Enfin, dans le cadre des récents bouleversements des relations internationales, Genève ne jouit plus de son quasimonopole de ville hôte des organisations internationales, qu'elle partage avec New York, et doit faire face à un climat de concurrence accrue.

\section{$\square$ Les réformes de l'ONU}

Elu en décembre 1996 à la fonction de secrétaire général des Nations Unies, le Ghanéen Kofi Annan est entré en fonction en janvier 1997 avec la lourde tâche de réformer l'organisation. En mars 1997, le nouveau secrétaire annonçait un premier train de mesures: la suppression de 1000 postes (sur 10'000) et l'annonce de la rationalisation des activités de 30 programmes, bureaux et fonds, en quatre secteurs (paix et sécurité, politiques économiques et sociales, développement, activités humanitaires). Le deuxième volet de la réforme, piloté par Maurice Strong, a été soumis aux Etats membres en juillet 1997. Lors d'une séance spéciale devant l'Assemblée générale Kofi Annan, en présentant son rapport «Rénover les Nations Unies: un programme de réformes», affirmait: «Les réformes que je propose sont ambitieuses. Il s'agit des plus vastes et radicales réformes opérées à l'ONU en cinquante-deux ans d'histoire. L'objectif en est simple: transformer l'organisation dans le sens d'une plus grande convergence des objectifs, d'une cohérence plus poussée des efforts et d'une plus grande souplesse afin de lui permettre de faire face aux exigences d'un monde de plus en plus dynamique et complexe.» Et de préciser que plus qu'un événement, un processus est engagé, «une révolution tranquille».

Les pressions externes et internes, venant des Etats membres comme des chefs d'agences, ont réussi à faire revoir les ambitions à la baisse. Ainsi, par exemple, l'UNICEF a fait un immense lobbying durant le printemps 1997 pour ne pas tomber sous la coupe du HCR et garder sa spécificité. L'accueil des réformes, plutôt chaleureux dans le monde occidental, a essuyé de sévères critiques dans les pays en développement qui craignent un démantèlement des programmes en leur faveur. Pour Maurice Bertrand, auteur d'une proposition de réforme de l'organisation dans les années 80 , la réforme de l'ONU est bien timide et n'est que le «camouflage d'une réduction d'effectifs»'. Selon lui, une véritable réforme devrait concerner en priorité la politique de maintien de la paix et de sécurité de

1. Journal de Genève, 17 juillet 1997 «La révolution tranquille de Kofi Annan». 
l'organisation, objectif premier de l'ONU. Il est vrai que le rapport n'évoque pas la réforme tant attendue du Conseil de sécurité, organe suprême de décision en matière de sécurité, qui compte aujourd'hui 15 pays membres, dont cinq permanents (Etats-Unis, Grande-Bretagne, France, Russie, Chine).

\section{LES PRINCIPAUX ÉLÉMENTS DE LA RÉFORME DE L'ONU}

ES RÉFORMES proposées par Kofi Annan devraient permettre à l'ONU de réaliser des économies en ratio-

nalisant les activités de l'organisation.

- Création d'un poste de vice-secrétaire général;

- suppression de 1000 postes de fonctionnaires sur les 10'000 existants aux secrétariats de New York, Genève et Vienne;

- réduction d'un tiers des dépenses administratives;

- création d'un fonds d'avances renouvelables doté d'un milliard de dollars, financé par des contributions volontaires, permettant à l'organisation de faire face à ses problèmes de trésorerie;

- centralisation à l'office de Vienne des efforts de la communauté internationale pour combattre le crime, la drogue et le terrorisme;

- les fonds et programmes de développement seront regroupés, incluant le PNUD, I'UNICEF et le FNUAP:

- le Département des affaires humanitaires (DAH) sera remplacé par un Office de coordination des secours d'urgence;

- création d'un Département du désarmement et de la réglementation chargé de la question de la réduction des armements et des armes de destruction massive;

- renforcement des activités relatives aux droits de l'Homme à Genève au sein d'une seule unité, I'Office du haut-commissaire pour les droits de l'Homme;

- déploiement plus rapide des opérations de maintien de la paix et autres opérations sur le terrain en renforçant la capacité de réaction rapide.

Source: site d'information sur les réformes de l'ONU: http://www. un. org/french/reform/

Le système des Nations Unies en quelques chiffres

L'ensemble du système des Nations Unies dans le monde, soit le Secrétariat et 25 autres agences spécialisées, ainsi que la Banque mondiale et le Fonds monétaire international, emploie environ 53'600 personnes. La totalité des dépenses de fonctionnement du système des Nations Unies s'est élevée à 18,2 milliards de dollars en 1996. Le budget de fonctionnement de 1'ONU est de 1,3 milliard de dollars par an.

Les sept pays dont la quote-part au budget de l'ONU est la plus élevée sont les Etats-Unis (25\%), le Japon (15,4\%), l'Allemagne (9\%), la France $(6,4 \%)$, le Royaume-Uni (5,3\%), l'Italie $(5,1 \%)$ et la Russie $(4,4 \%)$. La totalité de ces pays contribue pour plus du $66 \%$ du budget ordinaire de l'ONU. Les Etats-Unis d'Amérique sont le pays qui a les plus gros arriérés de contributions envers l'organisation (1,4 milliard de dollars). C'est également le pays qui a le plus grand nombre de fonctionnaires au Secrétariat et il détient le poste le plus élevé à l'UNICEF, au PNUD, au Programme alimentaire mondial, à l'Union postale universelle ainsi qu'à la Banque mondiale. Lors de la 52 ${ }^{\mathrm{e}}$ Assemblée générale de l'ONU qui s'est tenue en septembre 1997 à New York, le président Clinton a lié 
le remboursement de la dette américaine à l'élargissement du Conseil de sécurité, annonçant la décision unilatérale de Washington de diminuer sa quote-part de $25 \%$ à $20 \%$ pour les dépenses ordinaires et de $31 \%$ à $25 \%$ pour les opérations de maintien de la paix. La réaction des diplomates a été extrêmement virulente à l'égard des Etats-Unis; pourtant, quoi qu'en disent les 184 autres membres, les Américains sont en passe une fois de plus d'imposer leur point de vue.

\section{$\square$ Les contributions suisses au système des Nations Unies}

Conformément aux objectifs définis dans le rapport sur la politique extérieure de la Suisse dans les années 90, la participation aux activités des agences

\section{Tableau no 14}

Contributions de la Suisse au système des Nations Unies en millions de francs' (1996)

\begin{tabular}{lr}
\hline Association d'aide pour le développement (IDA) & 81.0 \\
\hline Banque internationale pour la reconstruction et le développement (BIRD) & 75.3 \\
\hline Programme des Nations Unies pour le développement (PNUD) & 60.5 \\
\hline Programme alimentaire mondial (PAM) & 32.6 \\
\hline Haut commissariat pour les réfugiés (HCR) & 29.2 \\
\hline Fonds asiatique pour le développement (FasD) & 25.1 \\
\hline Fonds africain pour le développement (FaD) & 21.2 \\
\hline Fonds des Nations Unies pour l'enfance (UNICEF) & 19.5 \\
\hline Fonds monétaire international (FMI) & 16.2 \\
\hline Organisation mondiale de la santé (OMS) & 13.7 \\
\hline Groupe consultatif pour la recherche agricole internationale (CGIAR) & 12.0 \\
\hline Société financière internationale (SFI) & 10.5 \\
\hline Fonds pour l'environnement mondial (FEM/GEF) & 10.2 \\
\hline Office des Nations Unies pour les réfugiés palestiniens (UNWRA) & 9.5 \\
\hline Fonds des Nations Unies pour la population (FNUAP) & 9.0 \\
\hline Centre du commerce international (CCI) & 7.0 \\
\hline Programme des Nations Unies pour l'environnement (PNUE) & 7.0 \\
\hline Département des Affaires humanitaires (DAH) & 6.6 \\
\hline UNESCO & 6.3 \\
\hline Conférence des Nations Unies pour le commerce et le développement (CNUCED) & 6.0 \\
\hline Organisation pour l'alimentation et l'agriculture (FAO) & 6.0 \\
\hline Union internationale des télécommunications (UIT) & 5.3 \\
\hline Bureau international du travail (BIT) & 5.2 \\
\hline Organisation des Nations Unies (participation au budget régulier) & 4.9 \\
\hline Agence internationale pour l'énergie atomique (AIEA) & $\mathbf{5 2 1 . 8}$ \\
\hline Autres & 37.2 \\
\hline Total & \\
\hline
\end{tabular}

1. Ces chiffres correspondent à l'ensemble des contributions suisses au système des Nations Unies. Ils incluent l'aide publique au développement (l'aide multi et multi-bi) qui passe par ces institutions.

Source: Département fédéral des affaires étrangères, Contributions suisses aux organisations internationales (document de travail). 
spécialisées de l'ONU constitue un des instruments les plus importants de la politique étrangère de la Suisse. La contribution helvétique au système des Nations Unies et aux institutions financières internationales s'est élevée pour 1996 à plus de 522 millions de francs. Bien que non-membre de l'ONU, la Confédération participe à de nombreux programmes et est membre à part entière de toutes les agences spécialisées. Comme Etat observateur, la Suisse a versé en 1996 une contribution d'environ 4,9 millions de francs au budget régulier de $\mathrm{l}^{\prime} \mathrm{ONU}^{2}$, qui correspond à $30 \%$ de la contribution que la Suisse aurait à payer si elle était membre de l'organisation. En effet, si la Suisse était membre de l'ONU sa participation serait de l'ordre de 15 millions de francs. Selon certaines sources, la totalité des contributions de la Suisse au système des Nations Unies place notre pays comme le sixième contributeur'.

\section{$\square$ Adhésion de la Suisse à l'ONU}

Onze ans après le refus du peuple et des cantons suisses (mars 1986) d'adhérer à l'ONU, la question est à nouveau à l'ordre du jour. Lors de la session parlementaire d'été 1997, une motion a été déposée par le conseiller national socialiste bâlois Remo Gysin demandant au Conseil fédéral de préparer l'adhésion de la Suisse, complétée par un postulat d'Andreas Gross, socialiste zurichois exigeant la rédaction d'un rapport sur les relations entre la Suisse et l'ONU dans un délai d'une année. Le 5 septembre 1997 le Conseil fédéral a répondu que l'adhésion de la Suisse à l'ONU tout comme celle à l'Union européenne font partie des objectifs définis dans le rapport du CF sur la politique extérieure de la Suisse dans les années 90, précisant que les relations avec l'UE gardent la priorité absolue. Le Conseil fédéral constate que la motion Gysin ne propose pas de calendrier, ce qui lui donne une certaine marge de manœuvre. Dans une première étape il élaborera un rapport conformément au postulat Gross lui permettant de réévaluer le calendrier pour la poursuite de la procédure d'adhésion. A noter que plus de 80 députés ont signé tant la motion que le postulat.

Quelques jours après cette prise de position, le Conseil fédéral accueillait le Secrétaire général de l'ONU à Berne pour sa première visite officielle. Kofi Annan s'est employé à calmer les inquiétudes liées aux conséquences pour Genève de la réforme de l'organisation internationale et a fermement souhaité «que la Suisse devienne membre à part entière d'ici quelque temps».

\section{$\square$ La Genève internationale}

En raison des profonds' changements de la situation géopolitique intervenus depuis la fin des années 80 , le choix de Genève comme siège des organisations internationales et lieu de grandes conférences internationales ne va plus de soi. En 1992, pour la première fois, la Suisse a ressenti de manière accrue la concurrence en matière d'accueil des OI lorsque la communauté internationale a donné sa préférence à La Haye comme siège de la nouvelle Organisation pour le contrôle des armes chimiques. En 1993, Genève avait eu le privilège d'accueillir les secrétariats intérimaires des trois conventions signées à Rio (changement climatique, biodiversité et désertification), mais n'a pas obtenu l'attribution défini-

2. DFAE, «Contributions suisses aux organisations internationales» (document de travail).

3. Nouveau Quotidien, 9 septembre 1997 «Kofi Annan encourage la Suisse à occuper enfin sa place aux Nations Unies». 
tive ni pour le climat (Bonn), ni pour la biodiversité (Montréal). En 1995, Genève a toutefois obtenu l'établissement du siège de l'OMC, mais en consentant d'importants efforts.

Pour répondre à cette situation nouvelle, un Groupe permanent conjoint Confédération-canton de Genève a été nommé en janvier 1995 et placé sous la direction du secrétaire d'Etat Jakob Kellenberger, du Département des affaires étrangères ${ }^{4}$. Le groupe s'est donné un triple objectif: réexaminer les priorités de la Genève internationale, déterminer une stratégie pour les années à venir et analyser les meilleurs moyens pour consolider la place de la ville dans un contexte de compétition croissante entre Etats hôtes.

La stratégie envisagée pour la Genève internationale s'appuie sur trois axes:

1) Consolidation du rôle de Genève dans les domaines où elle fait déjà figure de centre de coopération internationale. Les secteurs prioritaires englobent les activités dans les domaines de la protection de la vie (humanitaire, santé, environnement), des droits de l'homme, du travail et de l'économie, des connaissances et de la communication, et enfin de la promotion de la paix et du droit.

2) Renforcement de Genève comme centre de conférences internationales. Si le nombre de réunions internationales qui ont lieu chaque année à Genève est très important, la tenue de grandes conférences s'avère de plus en plus difficile à réaliser en raison d'un manque d'infrastructures adéquates; des efforts doivent être fournis pour remédier à cette situation.

3) Développement de l'information et de la communication. Ce point vise à faire prendre conscience à la population locale et suisse ainsi qu'aux décideurs politiques de tout niveau de l'importance de Genève comme ville hôte des organisations internationales pour la politique étrangère de la Suisse.

D'autre part le service du protocole de l'Etat de Genève, en collaboration avec la Confédération, a renforcé le service d'accueil du canton. Dans ce but, le Geneva Welcome Center a été inauguré en 1996 afin de faciliter l'installation et l'intégration à Genève des internationaux et l'accueil des organisations non gouvernementales.

Mission permanente de la Suisse près les organisations internationales

La Mission permanente de la Suisse près les organisations internationales à Genève est la charnière entre la Genève internationale et la politique étrangère suisse; son principal défi est d'assurer la coordination des diverses activités et d'assurer une collaboration optimale entre Berne, les autorités genevoises et les organisations internationales. La représentation suisse a deux tâches principales. D'une part, elle représente la Confédération auprès des organisations internationales, impliquant sa présence à plus de 1500 réunions multilatérales par an, soit plus de 15'000 séances à Genève. Cette fonction est assumée par la division multilatérale, forte d'une dizaine de personnes, qui couvre l'ensemble des relations multilatérales (désarmement, affaires humanitaires et droit de l'Homme, environnement, ainsi que les questions politiques, institutionnelles et budgétaires des organisations spécialisées). D'autre part, une vingtaine de collaborateurs de la division Etat hôte traitent de toutes les questions liées à la présence en Suisse des

4. Source: Rapport du Groupe permanent conjoint Confédération-Canton de Genève sur les priorités de la Genève internationale, 7 septembre 1995. 
organisations internationales, ainsi qu'à celle des 30'000 membres de la communauté internationale de Genève. Elle se charge également des problèmes que peuvent rencontrer plus de 80 '000 délégués venant chaque année à Genève pour participer aux réunions des organisations internationales.

\section{Mission permanente de la Suisse près l'OMC et l'AELE}

La Mission permanente de la Suisse près l'OMC et l'AELE, auprès de laquelle sont attachés une dizaine de collaborateurs, a pour tâche de participer aux réunions internationales ayant trait aux questions économiques.

\section{La Genève internationale: un nouveau rôle à définir}

Genève accueille plus d'une vingtaine d'organisations internationales intergouvernementales $(\mathrm{OI})$ et plus de 300 organisations non gouvernementales à caractère international, représentant plus de 30'000 postes de travail. Le secteur est le plus rémunérateur pour Genève, avant le secteur bancaire. Selon certaines estimations de la Mission suisse, les retombées économiques pour Genève et sa région sont d'environ 2,5 à 3 milliards de francs ${ }^{5}$. Pourtant la Genève internationale subit les contrecoups des restrictions budgétaires de l'ONU et, dans une moindre mesure, des réformes de l'organisation. Ainsi entre 1996 et 1998, ce sont plus de 2000 emplois qui ont été ou seront supprimés dans le secteur international (CERN, Secrétariat des Nations Unies, HCR, OMS, BIT, CNUCED). Seules l'Organisation mondiale du commerce et l'Organisation mondiale de la propriété intellectuelle créent de nouveaux postes de travail et engagent du personnel.

Les conséquences de la réforme proposée par Kofi Annan ne touchent que partiellement Genève, qui renforce sa position en matière de droits de l'Homme. En effet, le Haut-Commissariat de l'ONU pour les droits de l'Homme et le Centre de l'ONU pour les droits de l'Homme sont regroupés en un nouvel office placé sous l'autorité de Mary Robinson, haut-commissaire aux droits de l'Homme. Sous réserve d'une décision finale qui sera prise en mai 1998, lors de 1'Assemblée générale des Nations Unies, le Palais Wilson, restauré par la Confédération, deviendra la Maison des droits de l'Homme dans le courant de l'année 1998. Le Département des affaires humanitaires (DAH), qui partage ses activités entre New York et Genève, est rebaptisé «Office de coordination des affaires humanitaires». A New York sont concentrées les tâches de politique humanitaire, en lien notamment avec les décisions du Conseil de sécurité. A Genève seront décidés les choix de stratégies opérationnelles entre les acteurs concernés et la mise sur pied d'opérations pour répondre aux catastrophes naturelles.

Faisant face à une concurrence accrue avec d'autres villes, telles Vienne, Bonn, Montréal, La Haye ou Nairobi, Genève doit développer des stratégies de communication pour maintenir son importance au niveau international. Déçue de ne pas être devenue la capitale de l'environnement (les secrétariats du suivi de Rio se sont établis à Montréal pour la biodiversité et à Bonn pour le climat), Genève a cependant obtenu le siège de l'Organisation mondiale du commerce et s'affiche comme le centre international des droits de l'Homme. Pour les années à venir,

5. Discours de l'ambassadeur Walter B. Gyger, représentant permanent de la Suisse près les organisations internationales à Genève, à l'occasion de la séance extraordinaire du Conseil municipal de la Ville de Genève, le 15 septembre 1997. 
Genève cherche à s'imposer comme centre d'excellence en matière de télécommunication, s'appuyant sur de nombreuses organisations internationales présentes dans la ville et leaders en matière d'infocommunication.

\section{SOURCES}

«Rapport sur la politique extérieure de la Suisse dans les années 90», 29 novembre 1993, message 93.098.

International Geneva Yearbook - 1996 Organization and Activities of International Institutions in Geneva, vol. X and XI, Geneva, Eco'Diagnostic and Georg Editeur, 1996 et 1997.

Société pour le développement de l'économie suisse (SDES), Documentation, «Etat de la collaboration de la Suisse avec les organisations internationales, en particulier dans les domaines économique et social», $\mathrm{n}^{\circ} 29,15$ juillet 1997.

L'Agefi, 9 septembre 1997 «Genève n'a rien à craindre de la réforme de l'ONU», 17 juillet 1997 «Les activités humanitaires de l'ONU seront restructurées».

L'Hebdo, 3 juillet 1997 «ONU, le plan secret de Kofi Annan».

Journal de Genève, 6 juin 1996 «Les grands chantiers de la Genève internationale», 22 avril 1997 «Kofi Annan, depuis quatre mois dans ses nouvelles fonctions», 17 juillet 1997 «La révolution tranquille de Kofi Annan», 7 septembre 1997 «Le Conseil fédéral ouvre un œil sur l’ONU», 9 septembre 1997 «Kofi Annan à Berne».

La Liberté, 26 décembre 1996 «Genève subit le contrecoup des restrictions budgétaires».

Le Nouveau Quotidien, 9 septembre 1997 «Kofi Annan encourage la Suisse à occuper enfin sa place aux Nations Unies», 24 septembre 1997 «Washington lie le remboursement de ses dettes à l'ONU à l'élargissement du Conseil de sécurité».

Neue Zürcher Zeitung, 16. Dezember 1996 «Kofi Annan - Ein ruhiger Mann für gute Dienste», 12. Februar 1997 «Die Vereinten Nationen brauchen mehr als eine Führung», 2. Juli 1997 «Politische Ökonomie aus der Sicht der ONU», 4. Juli 1997 «Die Schweiz auf dem Uno-Parkett» von Botschafter Johannes J. Manz.

\section{SITES INTERNET}

Répertoire officiel des sites des organismes du système des Nations Unies (version française): http://www. unsystem. org/indfx. html

Site consacré à la Genève internationale: http://www. geneva-international. org/

\section{ADRESSE UTILE}

Mission permanente de la Suisse près les organisations internationales

rue de Varembé 9-11, case postale 194, 1211 Genève 20, tél. 022/749.24.24, fax 022/733.79.85.

\subsection{PROGRAMME DES NATIONS UNIES POUR LE DÉVELOPPEMENT (PNUD)}

Le Programme des Nations Unies pour le développement (PNUD) demeure le principal organe de programmation, de financement et de coordination du système onusien dans le domaine de la coopération technique. Il fournit une aide essentielle aux pays en développement et aux pays en transition pour l'élaboration de stratégies et de politiques de développement, le renforcement des capacités nationales de gestion, la coordination de l'aide extérieure ainsi que la promotion de la coopération Sud-Sud. Chaque année le PNUD publie le "Rapport mondial sur le développement humain». La Suisse soutient les activités du $P N U D$ par une importante contribution financière annuelle et une participation active aux travaux du Conseil d'administration.

\section{$\square$ Mandat et objectifs du PNUD}

Le PNUD a pour mission d'appuyer les efforts des pays en vue de parvenir à un développement humain durable, en les aidant à se doter des moyens propres à concevoir et à mettre en œuvre des programmes de développement dans les domaines de l'élimination de la pauvreté, de la création d'emplois et de moyens d'existence durables, de la promotion de la femme, et de la protection et de la régénération de l'environnement; il cherche en priorité à contribuer à l'élimination de la pauvreté. 
A travers le système des coordonnateurs résidants des Nations Unies, le PNUD s'efforce d'améliorer la cohésion entre les différentes entités du système onusien et s'emploie à renforcer la coopération internationale en matière de développement humain.

Outre ses programmes réguliers - pour la réalisation desquels le PNUD fait appel aux compétences des institutions spécialisées du système des Nations Unies, aux capacités propres des pays en développement (exécution nationale) ainsi qu'à des instituts de recherche travaillant dans différents domaines - le PNUD administre plusieurs fonds spéciaux (Fonds d'équipement des Nations Unies ${ }^{6}$, Fonds de développement des Nations Unies pour la femme, Fonds des volontaires des Nations Unies) et il participe à toute une série de programmes globaux (Fonds pour l'environnement mondial', Programme mondial de lutte contre le VIH/sida, Convention contre la désertification).

\section{Le financement du PNUD et l'utilisation des ressources}

Le financement du PNUD est en premier lieu assuré par des contributions générales (ressources de base) des pays participants. Les pays de l'OCDE fournissent environ 90\% de ces ressources qui s'élevaient en 1996 à 850 millions de dollars (contributions dites «volontaires»), la Suisse se situant, quant à elle, au huitième rang des pays donateurs avec une contribution de 56 millions de francs. Aux ressources générales s'est ajouté en 1996 un montant à peu près équivalent de contributions liées à des projets spécifiques; la tendance des deux dernières années montre une diminution des contributions générales et une augmentation des contributions à des projets/programmes spécifiques. Or, cette évolution pose problème car elle risque de mettre en danger la stabilité et la prévisibilité financières ainsi que la nature multilatérale de l'institution.

En ce qui concerne l'utilisation des ressources, $60 \%$ des contributions de base sont attribuées aux pays les moins avancés (PMA) et environ $90 \%$ aux pays dont le PNB par habitant est égal ou inférieur à 750 dollars par an.

\section{Session annuelle du Conseil d'administration du PNUD}

La session annuelle du Conseil d'administration du PNUD/FNUAP (session conjointe) s'est tenue à New York du 12 au 23 mai 1997, en respectant la tradition d'alternance biennale entre le siège du PNUD et Genève. La délégation suisse, conduite par Henri-Philippe Cart, vice-directeur de la DDC, est intervenue sur les deux sujets principaux à l'ordre du jour du conseil: les réformes du PNUD et le rapport annuel de l'Administrateur. Grâce à ses contributions financières et à l'intérêt qu'elle a toujours porté à la bonne marche de l'institution, la Suisse a, tout au long des années, pu jouer un rôle important au Conseil d'administration du PNUD (composé de 36 membres représentant les pays tant développés qu'en développement); par un système de rotation elle en a été membre à part entière pour la période 1996-97 et elle le sera à nouveau pour 1999-2000. Avec de nombreux autres pays (donateurs et récipiendaires), la Suisse reconnaît

6. La Suisse a contribué au FENU pour 2,5 millions de francs en 1996.

7. En coopération avec la Banque mondiale et le Programme des Nations Unies pour l'environnement, le PNUD est l'un des gestionnaires du FEM. Ce fonds, doté de 2 milliards de dollars, aide les pays à mettre sur pied des plans d'action nationaux pour faire front aux menaces pour l'ensemble de la planète que constituent l'appauvrissement de la couche d'ozone, le réchauffement de la planète, la perte de diversité biologique ou la pollution des eaux internationales. La première contribution de la Suisse au FEM s'est élevée à 64 millions de francs. 
au PNUD un rôle clé, tant au sein du système des Nations Unies que dans le cadre de la coopération au développement en général.

\section{Les réformes du PNUD}

Les activités opérationnelles du système des Nations Unies traversent une phase difficile. Les principaux fonds et programmes du système, et tout particulièrement le PNUD, sont sans cesse chargés de nouvelles obligations et responsabilités. Preuve en sont, entre autres, les tâches qui leur ont été confiées en vue de la mise en œuvre des programmes et plans d'action des grandes conférences internationales de ces dernières années (sommets sur les enfants, l'environnement, le développement social et l'alimentation; conférences sur la population, les femmes et l'habitat). Or les ressources de base dont disposent les fonds et programmes en question tendent à diminuer (du moins en termes réels).

Le PNUD a entrepris, depuis la mi-1996, un processus de restructuration interne qui doit lui permettre de mieux accomplir sa mission et de renforcer son rôle en matière de développement humain. Dans cette perspective, toute une série de mesures ont été entreprises ou le seront très prochainement (décentralisation de l'organisation par un renforcement des bureaux extérieurs et du système des coordonnateurs résidants, consolidation des évaluations et du suivi des programmes, création de centres de ressources sous-régionaux, etc.). La Suisse, qui soutient la décentralisation du PNUD, espère que toutes ces mesures qui vont dans la direction d'une focalisation accrue de l'activité du PNUD sur le terrain permettront à l'organisation de fournir des services et appuis de haute qualité, répondant à des besoins et à des priorités spécifiques définis par les pays concernés.

\section{Rapport annuel de l'Administrateur pour 1996}

Dans son rapport, l'administrateur, James Gustave Speth, a mis en exergue les efforts accomplis par l'organisation dans sa lutte contre la pauvreté et la promotion de la femme. Il a également dressé un tableau de la situation financière, rappelant que les contributions de base des pays donateurs constituent l'assise financière du PNUD. Or les contributions d'importants pays donateurs semblent plafonner. La délégation suisse, préoccupée par la baisse des ressources de base, a souligné la nécessité de garder un niveau de ressources à 1 milliard de dollars par an et invité les grands pays à reprendre leur part de financement traditionnelle destinée aux opérations du PNUD. Pour sa part, la Suisse a participé au financement de l'organisation et de ses programmes associés pour un montant de 58 millions de francs suisses en 1996. Cette contribution multilatérale de la Confédération est la plus importante fournie par la Suisse aux agences spécialisées des Nations Unies.

\section{$\square$ Bref aperçu des activités du PNUD}

Si l'année écoulée a été caractérisée par le processus de réformes internes mentionnées ci-dessus, le PNUD a néanmoins poursuivi sa tâche principale qui consiste à appuyer les pays dans leurs efforts pour réaliser un développement humain durable en mettant tout particulièrement l'accent sur la lutte contre la pauvreté.

\footnotetext{
8. Pour connaître le détail de l'aide publique multilatérale au développement de la Confédération, se référer à la partie «Statistiques», p. 347.
} 


\section{Lutte contre la pauvreté}

Malgré des acquis significatifs dans la croissance économique et une amélioration générale du niveau de vie durant les trois dernières décennies, environ $20 \%$ de la population mondiale continue à vivre en dessous du seuil de pauvreté. Généralement, elle n'a pas accès aux bases mêmes d'une vie décente - nourriture suffisante, eau potable, soins médicaux fiables, logement adéquat, éducation, opportunités de formation et d'activités génératrices de revenus. Trop souvent, les pauvres sont impuissants à changer leur situation parce qu'ils ont peu de chance de participer aux prises de décision qui affectent leur vie.

Lors du Sommet mondial pour le développement humain, communément appelé le Sommet social, qui s'est tenu à Copenhague au Danemark en mars 1995, les chefs d'Etat et les principaux dirigeants de plus de 180 pays ont reconnu l'aggravation du problème de la pauvreté globale et se sont engagés à agir pour la combattre. Suite aux décisions du Sommet social, le Conseil d'administration a décidé de faire de l'élimination de la pauvreté la priorité essentielle du PNUD.

Outre la publication du Rapport mondial sur le développement humain (voir cidessous), le PNUD a aidé plus de 50 pays à établir un premier rapport national sur le développement humain; ces documents ont dans bien des cas servi de base de concertation entre les différents acteurs locaux ainsi que de point de départ pour l'élaboration de politiques et de stratégies de lutte contre la pauvreté à l'échelon national.

Quant aux activités de terrain à proprement parler, la direction du PNUD a focalisé davantage ses nouveaux programmes de pays sur des domaines ayant un impact direct sur la réduction de la pauvreté, à savoir, la création d'emplois et d'autres formes de revenu ainsi que la promotion de microfinancements, l'égalité des sexes et la promotion de la femme dans les domaines économique et politique, la protection et la régénération de l'environnement.

L'activité du PNUD en 1996-97 a été en outre caractérisée par les éléments suivants:

\section{- Le renforcement du système des coordonnateurs résidants}

Les tâches de coordination du PNUD et de ses bureaux externes ne se limitent plus aujourd'hui à la gestion des «programmes par pays», mais couvrent également dans bien des cas (pays en crise et en situation d'urgence) des opérations d'aide d'urgence, de reconstruction et de maintien de la paix.

\section{- Le suivi des grandes conférences internationales}

Le PNUD a continué à aider de nombreux pays qui en ont fait la demande à élaborer des programmes nationaux de mise en œuvre des différents plans d'action internationaux. Il convient de signaler en particulier les efforts déployés dans un certain nombre de pays par les coordonnateurs résidants pour constituer des équipes de pays (interagences et pluridisciplinaires) chargées du suivi de ces conférences.

\section{- Pays en crise et en situation particulière}

En étroite collaboration avec les pays concernés et avec les partenaires au sein du système des Nations Unies, le PNUD a lancé toute une série d'opérations por-

9. Un dossier d'information, Eliminer la pauvreté, publié par le PNUD en juin 1996 présente les stratégies de l'organisation dans ce domaine, ainsi que ses réalisations dans différents pays. 
tant notamment sur la démobilisation et la réintégration des combattants, le déminage, l'appui à l'organisation d'élections, la reconstruction des zones de retour des personnes déplacées, la reconstruction de zones après des catastrophes naturelles, le rétablissement des capacités gouvernementales en matière de planification et de suivi des programmes.

\section{«Rapport mondial sur le développement humain 1997»}

Le Rapport mondial sur le développement humain 1997 met en lumière l'ampleur, la gravité et la complexité de la pauvreté à l'échelle mondiale. Il propose une stratégie pratique (en six points) en vue d'éradiquer ce fléau:

$\checkmark$ donner les moyens aux hommes et aux femmes les plus pauvres d'améliorer leur sort (éducation, accès à la terre et aux crédits, sécurité de logement);

- favoriser l'égalité entre les hommes et les femmes (prise en compte du point de vue des femmes dans les décisions au sein de la famille, aux niveaux national et international);

- orienter la croissance économique pour qu'elle soit en premier lieu favorable aux plus pauvres (préférence donnée aux projets faisant appel à la maind'œuvre, création d'un environnement stimulant pour l'agriculture à petite échelle, aux microentreprises et au secteur informel);

- maîtriser la mondialisation afin de limiter l'accroissement du fossé qui sépare les gagnants et les perdants (meilleure répartition des investissements étrangers, réévaluation des règles commerciales pour favoriser les pays les plus pauvres - réduction des barrières douanières contre les produits du Sud et diminution des subventions agricoles au Nord);

- créer un environnement politique qui favorise la volonté de s'attaquer de front au problème de la pauvreté (responsabiliser les pauvres pour qu'ils interviennent politiquement afin d'influer sur leurs conditions de vie, encourager les $\mathrm{ONG}$ à participer à la définition des politiques visant l'éradication de la pauvreté);

- coordonner des mesures au niveau international (réduction de la dette, augmentation de l'aide publique au développement, facilité l'accès des marchés mondiaux).

Le rapport redéfinit la notion de pauvreté, en précisant qu'elle doit être abordée dans la totalité de ses dimensions, et pas seulement sous l'angle du revenu. Pour permettre cette analyse, le PNUD a développé un nouvel indicateur (voir encadré) qui combine des aspects essentiels de la pauvreté et révèle d'intéressants contrastes avec la pauvreté monétaire. Premier constat, la pauvreté monétaire comme la pauvreté sont des phénomènes très répandus, qui concernent entre un tiers et un quart des habitants du monde en développement. En Afrique subsaharienne et en Asie du Sud la pauvreté monétaire et la pauvreté humaine sont les plus répandues puisqu'elles touchent $40 \%$ de la population. Les pays arabes, quant à eux, ont accompli des progrès remarquables dans la réduction de la pauvreté monétaire, qui a été ramenée à $4 \%$ de leur population, mais beaucoup reste à faire dans la lutte contre la pauvreté humaine qui frappe encore $32 \%$ de leurs habitants.

La Suisse recule d'une place dans le classement annuel du rapport du PNUD. Elle cède le $15^{\mathrm{e}}$ rang pour prendre le $16^{\mathrm{e}}$ rang mondial. En tenant compte des inégalités homme-femme, elle ne se situe qu'au $20^{\circ}$ rang. 


\section{LES INDICATEURS DU PNUD}

L'indicateur du développement humain (IDH) mesure le niveau moyen auquel se trouve un pays donné selon trois critères essentiels: l'espérance de vie, le niveau d'éducation et le PIB réel par habitant (mesuré en PPA ou parités de pouvoirs d'achat).

L'indicateur de la pauvreté humaine (IPH) s'attache aux déficits rencontrés dans trois domaines essentiels de l'existence humaine (déjà pris en compte dans I'IDH). Les variables utilisées sont le pourcentage d'individus risquant de décéder avant l'âge de 40 ans, le pourcentage d'adultes analphabètes, le pourcentage d'individus n'ayant pas accès aux services de santé ni à l'eau potable, et le pourcentage d'enfants de moins de 5 ans victimes de malnutrition.

L'indicateur sexospécifique du développement humain (ISDH) s'attache aux potentialités humaines essentielles en se fondant sur les mêmes variables que I'IDH, corrigées en fonction des inégalités sociologiques entre les sexes. En fait I'ISDH correspond à un IDH révisé à la baisse, en fonction des inégalités entre femmes et hommes.

L'indicateur de la participation de femmes (IPF) indique si les femmes sont en mesure de prendre une part active à la vie politique et économique. II se concentre sur la participation et mesure les inégalités sociologiques entre les sexes et en termes de représentation et de pouvoir de décision dans certains domaines clés de la sphère économique et politique. II diffère ainsi de l'ISDH, qui constitue un indicateur des inégalités entre les sexes sur le plan des potentialités de base.

$\llbracket$ ASTM 1996, «Sommet mondial pour le développement social 1995», pp. 60-65.

\section{SOURCES}

United Nations Development Programme, UNDP 1996-1997 Annual Report, UNDP, October 1997.

Nations Unies, Conseil d'administration du Programme des Nations Unies pour le développement et du Fonds des Nations Unies pour la population, rapport du Directeur exécutif PNUD pour 1996, session annuelle du 12 au 23 mai 1997, New York (DP/1997/16).

Programme des Nations Unies pour le développement, Rapport sur le développement humain 1997, Paris, Ed. Economica, 1997.

Programme des Nations Unies pour le développement, dossier d'information Eliminer la pauvreté, PNUD, juin 1996.

Choix, la revue du développement humain, PNUD, avril et juillet 1997.

L'Agefi, 12 juin 1997 «La mondialisation de l'économie est un danger pour les pays pauvres», 14 juillet 1997 «A contresens».

La Liberté, 13 juin 1997 «Développement humain, la Suisse recule d'une case».

Mosquito, «Armut ist vermeidbar», Nr. 7/97.

Neue Zürcher Zeitung, 13. Juni 1997 «Einseitiges Uno-Manifest zur Armutsbekämpfung».

\section{SITE INTERNET}

Le site officiel du PNUD: http://www. undp. org./ 


\subsection{FONDS DES NATIONS UNIES POUR LA POPULATION (FNUAP)}

En septembre 1994, la Conférence internationale sur la population et le développement (communément appelée conférence du Caire) a publié un programme d'action, fruit d'un consensus historique, par lequel tous les pays s'engagent à réaliser au cours des vingt prochaines années un ensemble d'objectifs concrets dans le domaine de la santé, de l'amélioration de la condition de la femme et du développement social. Le Fonds des Nations Unies pour la population (FNUAP) a joué un rôle très important dans la préparation et le déroulement de ce sommet. Depuis lors, le FNUAP a opéré des changements institutionnels et révisé ses principes directeurs afin de mieux intégrer et mettre en cuvre les décisions prises au Caire. La Suisse fait partie du Conseil d'administration conjoint $P N U D / F N U A P$ et soutient financièrement le FNUAP par des contributions générales annuelles.

\section{$\square$ Mandats et objectifs du FNUAP}

Le Fonds des Nations Unies pour la population (FNUAP) fondé en 1967 a pour tâche d'intégrer les objectifs démographiques dans les stratégies internationales et nationales de développement. Le Fonds a pour organe directeur le Conseil d'administration du PNUD/FNUAP. Nafis Sadik en est la directrice exécutive depuis 1987.

Le mandat du FNUAP a été défini en 1973 par le Conseil économique et social des Nations Unies; il porte sur:

- le développement des connaissances relatives à la démographie et à la santé génésique;

- la mise en place de centres de planification familiale;

- la sensibilisation des pays développés et en développement aux problèmes démographiques et aux stratégies susceptibles de les résoudre;

- le soutien, pour les pays qui le demandent, en vue de faire face aux problèmes de population avec les moyens les mieux adaptés selon les situations.

\section{$\square$ Financement}

Le FNUAP est financé presque totalement par des contributions générales des pays donateurs traditionnels et non par le budget ordinaire de l'ONU. En 1996, les ressources du FNUAP se sont élevées à 310 millions de dollars (environ), contributions de 95 pays, dont la plupart sont des pays en développement, ainsi que d'autres organismes multilatéraux (Banque mondiale). Mais le financement le plus important provient des pays industrialisés: les 14 principaux pays donateurs ont participé pour $98 \%$ au financement du fonds, soit pour un montant de 296 millions de dollars. Cependant, le retard dans l'acquittement de contributions annoncé par un tiers des Etats a posé d'importants problèmes de trésorerie à l'institution. Pour sa part, la Suisse a contribué au FNUAP pour un montant de 10 millions de francs en 1997.

\section{$\square$ Mise en æuvre du plan d'action de la Conférence sur la population}

Parmi les activités essentielles du FNUAP figure la mise en œuvre du plan d'action de la Conférence du Caire (promotion de l'égalité et l'équité entre les sexes, 
renforcement des moyens d'action des femmes, élimination de toutes les formes de violence à leur égard et meilleure maîtrise de leur propre fécondité). Le FNUAP finance des programmes encourageant la santé génésique et l'éducation des femmes afin de renforcer leur position au sein de la famille et de la société. Il encourage leur accès à des activités rémunératrices qui leur permettent d'améliorer leur bien-être ainsi que celui de leur famille. Enfin, il favorise un recours plus large à la planification familiale, visant une réduction des taux de fécondité et de mortalité infantile. La mise en œuvre du programme d'action de la conférence du Caire a nécessité une profonde réforme de l'institution, dans les structures institutionnelles, les principes directeurs, les procédures opérationnelles et les activités menées en coopération avec d'autres agences.

\section{$\square$ Session annuelle du Conseil d'administration conjointe PNUD/FNUAP}

La session annuelle du Conseil d'administration du PNUD et du FNUAP (session conjointe) s'est tenue à New York du 12 au 23 mai 1997. La Suisse a été membre du Conseil d'administration jusqu'en décembre 1997. La délégation suisse était conduite par Henri-Philippe Cart, vice-directeur de la DDC. A l'ordre du jour figurait l'analyse de divers points du rapport annuel de la Directrice exécutive.

Dans son rapport annuel pour 1996 Nafis Sadik passe en revue les trois domaines principaux d'intervention du FNUAP définis lors de la conférence du Caire. L'objectif principal concerne la promotion de la «santé de la reproduction» qui a trait à la planification familiale et à l'hygiène sexuelle. Cette notion remplace et complète la notion controversée du «contrôle des naissances» qui est apparue aux participants de la conférence du Caire comme une notion très autoritaire et simpliste. Concernant ce point un accent particulier a été donné à la santé des adolescents en matière de reproduction. A noter également que la Journée mondiale de la population de 1997 leur était consacrée. Nafis Sadik a rappelé qu'aujourd'hui les jeunes de 15 à 24 ans n'ont jamais été aussi nombreux puisqu'ils représentent un milliard d'individus. Et de constater que dans leur très grande majorité les jeunes veulent en savoir plus; ils souhaitent des informations sur la sexualité et la santé sexuelle, et savoir comment se protéger pour éviter une grossesse non désirée ou une maladie transmissible comme le sida. Face à cette demande N. Sadik a insisté pour que les promesses soient tenues, en particulier les engagements qu'ont pris tous les pays à la conférence du Caire en $1994^{10}$.

Un deuxième volet d'activités est le soutien aux «stratégies relatives à la population et au développement» qui ont pour objectif d'aider les gouvernements à tenir compte des questions intéressant la population dans leurs politiques. Un soutien technique leur est apporté pour faciliter le recensement, l'analyse et l'utilisation des statistiques et des données démographiques.

Les «activités de mobilisation», enfin, visent à rappeler aux gouvernements ainsi qu'aux organisations internationales et non gouvernementales le rôle que chaque partenaire doit jouer dans la prise de conscience des questions liées à la population et au développement. Dans cette perspective, le FNUAP valorise les droits en matière de procréation, l'égalité entre les sexes, la responsabilité masculine 
ainsi que l'autonomie et la promotion des femmes partout dans le monde. Il estime que tout couple et tout individu ont le droit de décider librement du nombre de leurs enfants et de l'espacement entre les naissances et que, pour ce faire, ils doivent disposer d'informations et de moyens à cet effet.

\section{$\square$ Etat de la population mondiale 1997}

Le rapport sur l'Etat de la population dans le monde 1997 est consacré à l'exercice des droits en matière de sexualité et de reproduction. Il présente une synthèse des accords internationaux relatifs à ces droits, qui portent sur:

- le droit à la santé en matière de sexualité et de reproduction;

- le droit de prendre des décisions en matière de reproduction (le choix volontaire du mariage, de la décision de fonder une famille, du nombre d'enfants ainsi que l'accès à l'information et aux moyens requis pour exercer un choix volontaire);

- le droit à l'égalité et à l'équité pour les hommes et pour les femmes;

- le droit à la sécurité en matière de sexualité et de reproduction (y compris le droit de ne pas être soumis à la violence sexuelle et à la coercition dans ce domaine).

Malgré la reconnaissance de ces droits, le rapport dresse un bilan alarmant de la situation en la matière:

- 585'000 femmes - une par minute - meurent chaque année des suites d'une grossesse; dans la plupart des cas il s'agit de femmes vivant dans les pays en développement;

口 entre 120 et 150 millions de femmes souhaitent limiter le nombre de leurs grossesses ou les espacer, mais n'ont pas toujours les moyens de le faire;

- chaque année, 70'000 femmes meurent des suites d'un avortement pratiqué dans de mauvaises conditions;

- on dénombre 120 millions de femmes qui ont subi la mutilation de l'appareil génital, et 2 millions courent ce risque chaque année;

- chaque année 2 millions de fillettes sont livrées au commerce du sexe;

- le viol et la violence sexuelle se répandent;

- la violence familiale est chose courante dans la plupart des sociétés.

Pour des millions de femmes, le respect des droits en matière de sexualité et de reproduction est une question de survie. Pour des millions d'autres cela leur permettrait de vivre à l'abri de la maladie et de la coercition sexuelle. Face à ce constat, des progrès très importants doivent être réalisés; le rapport établit une liste des mesures à mettre en œuvre. La satisfaction des besoins mondiaux de soins de santé coûtera chaque année, selon la conférence du Caire, 17 milliards de dollars jusqu'en l'an 2000.

\section{Des parlementaires suisses en mission au Vietnam}

Répondant à l'invitation de la Fédération internationale pour le planning familial (IPPF) et de l'Association suisse de planning familial et d'éducation sexuelle (ASPFES), dix parlementaires suisses ${ }^{11}$, autrichiens et finlandais se sont rendus

11. La délégation suisse était emmenée par l'ancienne conseillère nationale Leni Robert, présidente de l'ASPFES, et était composée de Remo Gysin (PS/BS), Pia Hollenstein (PES/SG), Liliane Maury Pasquier (PS/GE), Marc Suter (PRD/BE) et Rose-Marie Zapfl-Helbling (PDC/ZH). 
en avril 1997 au Vietnam pour s'informer de l'état et des besoins des centres de planning familial et de santé reproductive. Dans une résolution finale qu'ils ont remise au conseiller fédéral Flavio Cotti en mai 1997, les parlementaires demandent que l'aide aux problèmes de reproduction soit intégrée à la coopération au développement.

ASTM 1995, «Conférence internationale sur la population et le développement 1994», pp. 70-75.

\section{SOURCES}

Nations Unies, Conseil d'administration du Programme des Nations Unies pour le développement et du Fonds des Nations Unies pour la population, rapport du Directeur exécutif FNUAP pour 1996, session annuelle du 12 au 23 mai 1997, New York (DP/FPA/1997/10).

Fonds des Nations Unies pour la population, Etat de la population mondiale 1997, New York, FNUAP, mai 1997.

Espace Femmes International, Newspack Femmes et Développement, paraît dix fois par an, EFI, 1997.

Journal de Genève, 29 mai 1997 «L'ONU publie un rapport sur la santé de la population».

Nouveau Quotidien, 29 mai 1997 «Des parlementaires suisses en mission au Vietnam».

Neue Zürcher Zeitung, 29. Mai 1997 «Neuer Aufruf der Uno zur Emanzipation der Frau».

SITE INTERNET

Fonds des Nations Unies pour la population: http://www. unfpa. org./

\subsection{SUIVI DE LA CONFÉRENCE DE PÉKIN (FEMME ET DÉVELOPPEMENT) EN SUISSE}

En septembre 1995 s'est tenue à Pékin la $4^{e}$ Conférence mondiale des Nations Unies sur les femmes, avec pour thème «Egalité, développement et paix». L'objectif de cette conférence était de faire un état des lieux de la situation dans laquelle vivent les femmes de par le monde et de fixer des objectifs communs afin de guider la communauté internationale et l'ensemble des Etats dans leurs efforts pour faire avancer l'égalité entre femmes et hommes. Le Programme d'action décidé lors de la conférence doit être mis en æuvre dans les politiques nationales. La Suisse a établi son agenda politique pour la mise en application du programme.

Afin de rendre accessible au public le Programme d'action, le Bureau fédéral de l'égalité entre femmes et hommes et la Direction du développement et de la coopération ont publié, dans un langage simple et direct, une brochure ${ }^{12}$ résumant les décisions prises à Pékin. La Déclaration de Pékin est suivie de 12 chapitres $^{13}$ présentant les thèmes de la conférence. Chacun des chapitres présente le sujet traité dans le contexte international et suisse, et est complété par des statistiques pertinentes. Suivent les objectifs à atteindre au niveau tant international que suisse.

12. Bureau fédéral de l'égalité entre femmes et hommes et Direction du développement et de la coopération, Résumé du Programme d'action - Conférence mondiale sur les femmes - Pékin, Berne, novembre 1996. Disponible à l'Office central fédéral des imprimés et du matériel, 3003 Berne.

13. Pauvreté, éducation et formation, santé, violence à l'égard des femmes, conflits armés, économie, prises de décision, mécanismes institutionnels chargés de favoriser la promotion de la femme, droits fondamentaux, médias, environnement, petite fille. Chacun de ces chapitres est abordé sous l'angle de la situation des femmes. 


\section{Rédaction du plan d'action suisse}

La mise en œuvre sur le plan national du Programme d'action de Pékin nécessite une traduction des objectifs généraux à la situation de la Suisse. L'élaboration du plan national a été confiée à un groupe interdépartemental de l'administration fédérale chargé de faire un recensement des actions et intentions des différents offices concernés. Le plan d'action sera soumis aux organisations non gouvernementales avant d'être officiellement accepté. Les mesures proposées concerneront la situation intérieure et la politique extérieure de la Suisse. La consultation et la finalisation du document doivent se poursuivre durant le printemps 1998 et il sera vraisemblablement disponible en automne.

\section{Rapport du Conseil fédéral sur son engagement}

en faveur des femmes dans le cadre de sa politique étrangère

Pour répondre à une interpellation de Judith Stamm, le Conseil fédéral a rédigé un rapport ${ }^{14}$ présentant l'engagement de la Suisse en faveur des femmes dans sa politique étrangère (définie dans le rapport sur la politique extérieure de la Suisse dans les années 90). Le rapport se concentre plus spécifiquement sur quatre domaines prioritaires: égalité, paix, droits de l'homme et développement. Cependant, le Conseil fédéral rappelle que dans la politique d'aide au développement et dans les projets de coopération, une priorité est mise sur un développement équilibré entre les femmes et les hommes (politique transversale).

\section{Ratification de la Convention \\ sur l'élimination des discriminations à l'égard des femmes}

La Convention des Nations Unies de 1979 sur l'élimination de toutes les formes de discrimination à l'égard des femmes concrétise l'interdiction de discrimination dans tous les domaines de la vie. Elle oblige les Etats à prendre, dans les domaines politique, économique, social et culturel, des mesures devant conduire à l'élimination des discriminations dont les femmes sont victimes. Traité de droit international juridiquement contraignant auquel 154 Etats sont déjà parties, la convention représente une contribution, sur le plan international, en faveur de l'égalité homme-femme. Elle complète les conventions déjà ratifiées par la Suisse en matière de protection des droits de l'Homme, en particulier les deux pacte $^{15}$ des droits de l'Homme, auxquels la Suisse a adhéré en 1992.

La ratification de la convention a été proposée par le Conseil fédéral en août 1995 et approuvée par les Chambres fédérales lors de la session d'automne 1996. Le Conseil fédéral a signé la convention en 1987 déjà, mais il avait décidé de faire dépendre la ratification de mesures législatives à prendre en droit suisse. Le $1^{\text {er }}$ juillet 1996 la loi sur l'égalité est entrée en vigueur, instrument essentiel pour la réalisation dans les faits de l'égalité entre femmes et hommes dans la vie professionnelle.

14. Département fédéral des affaires étrangères, Rapport du Conseil fédéral sur son engagement en faveur des femmes dans le cadre de sa politique étrangère, Berne, 6 novembre 1996. Disponible auprès du DFAE, tél. 031/322.35.55.

15. Les deux pactes internationaux du 19 décembre 1966 relatifs aux droits économiques, sociaux et culturels (Pacte I) ainsi qu'aux droits civils et politiques (Pacte II) établissent un catalogue international de droits et libertés appartenant à tous les individus, contraignants pour les Etats qui les ratifient. 
L'instrument de ratification a été déposé par la Suisse en mars 1997 auprès du Secrétaire des Nations Unies à New York et est entré en vigueur trente jours après le dépôt. Trois réserves ont toutefois été formulées par la Suisse, la première concernant la situation des femmes au sein de l'armée, la seconde concernant le choix du nom de famille, la troisième concernant les couples dont le mariage dépend de l'ancien droit matrimonial. Les deux premières réserves pourraient perdre leur justification suite à une révision législative. Quand à la troisième, elle ne s'imposera plus au fil des ans.

[D ASTM 1996, "4 4 Conférence mondiale sur les femmes », pp. 66-74.

\section{SOURCES}

Bureau fédéral de l'égalité entre femmes et hommes et Direction du développement et de la coopération, Résumé du Programme d'action - Conférence mondiale sur les femmes - Pékin, Berne, novembre 1996.

Département fédéral des affaires étrangères, Rapport du Conseil fédéral sur son engagement en faveur des femmes dans le cadre de sa politique étrangère, Berne, 6 novembre 1996.

«Message relatif à la Convention de 1979 sur l'élimination de toutes les formes de discrimination à l'égard des femmes du 23 août 1995 », message 95.060 .

Bureau fédéral de l'égalité entre femmes et hommes, Dossier d'information sur la $4^{e}$ Conférence mondiale de l'ONU sur les femmes, Berne, 1995.

Helvetas, Partenaires, dossier «De la parole aux actes: les femmes s'engagent», n 147, février 1997.

\subsection{ORGANISATION INTERNATIONALE DU TRAVAIL}

Lors de la $1^{r e}$ Conférence ministérielle de l'OMC, qui s'est tenue en décembre 1996 à Singapour, Michel Hansenne, directeur général du BIT, s'était vu retirer son invitation à participer à la conférence. La question de l'introduction de normes sociales dans les échanges internationaux avait fait l'objet de vifs débats. L'OMC, souhaitant éviter un trop grand antagonisme Nord-Sud, n'avait pas inscrit ce thème dans l'agenda de ses travaux, mais au contraire avait réaffirmé le rôle de l'OIT dans l'établissement de normes sociales et leur contrôle. La Déclaration de Singapour a donné une nouvelle légitimité à l'OIT. Dans cet élan, le Directeur général a fait d'ambitieuses propositions afin de renforcer le respect des normes fondamentales des travailleurs. Le travail des enfants a été également un des thèmes importants de l'OIT en 1997 et le demeurera pour les années à venir.

\section{$\square$ Conférence internationale du travail, juin 1997}

La conférence a accueilli deux hôtes d'honneur, le roi Hussein de Jordanie et le directeur de la Banque mondiale, James Wolfensohn. Ce dernier a relevé les collaborations passées entre les deux institutions, mais en soulignant que les relations n'avaient pas été suffisamment étroites pour permettre de relever le type de défis auxquels le monde est confronté. Il a encouragé de nouvelles collaborations et conclu en disant que «les objectifs de l'OIT et de la banque sont indissolublement liés. La façon dont nous travaillons est certes différente, mais nous sommes manifestement complémentaires ${ }^{16}$.

16. OIT, Communiqué de presse, BIT/97/16, 12 juin 1997, «Le président de la Banque mondiale prend la parole devant la Conférence internationale du travail». 


\begin{abstract}
A CONFÉRENCE internationale du travail se réunit chaque année au mois de juin. Elle constitue une tribune mondiale où sont discutés les problèmes du travail et les questions sociales. Elle établit les normes internationales du travail et les objectifs généraux de l'organisation. Tous les deux ans la Conférence adopte le programme biennal de I'OIT et son budget financé par 174 Etats membres. Chaque Etat membre a le droit de se faire représenter à la Conférence par quatre délégués: deux gouvernementaux, un des travailleurs et un des employeurs; chacun d'eux est libre de s'exprimer et d'y voter en toute indépendance.
\end{abstract}

Date et lieu La conférence s'est tenue au siège de I'OIT à Genève du 3 au 19 juin 1997.

Thèmes de la conférence

- Examen du rapport du Président du Conseil d'administration sur les travaux de l'année écoulée:

- examen et adoption du programme et du budget pour l'exercice 1998-99;

$\checkmark$ discussion sur le rapport du Directeur général consacré cette année à l'action normative du BIT à I'heure de la globalisation;

$\checkmark$ révision de la convention n ${ }^{\circ} 96$ sur les bureaux de placement privés;

$\checkmark$ premières discussions sur deux conventions, l'une concernant les conditions générales pour stimuler la création d'emplois dans les petites et moyennes entreprises (en vue de l'élaboration d'une recommandation pour 1998), I'autre concernant le travail en soustraitance (en vue de l'élaboration d'une convention pour 1998);

• amendement constitutionnel permettant l'abrogation de conventions internationales sur le travail obsolètes.

Participants

La conférence a réuni 2025 représentants des gouvernements, des employeurs et des travailleurs de 158 Etats, sur les 174 Etats membres. Un nombre record de 143 délégations étaient conduites par des ministres.

Délégation suisse

La délégation suisse, forte d'une vingtaine de membres, était dirigée par Jean-Luc Nordmann, secrétaire d'Etat, directeur de I'OFIAMT.

\title{
Discussion sur un renforcement des normes
}

Lors de la Conférence ministérielle de l'OMC tenue à Singapour, les ministres ont rappelé le principe des avantages comparatifs entre les pays, en particulier des pays à bas salaires, et insisté pour qu'il ne soit pas remis en question. Parallèlement, ils ont réaffirmé leur volonté de veiller à l'observation des normes fondamentales du travail internationalement admises et ont reconnu que l'OIT est l'organe compétent pour établir ces normes et les faire respecter.

Pour donner suite à la conférence de Singapour, le Directeur général du BIT a rédigé un important rapport, L'action normative de l'OIT à l'heure de la mondialisation $^{17}$, qui a fait l'objet d'un débat lors de la conférence. Dans ce rapport Michel Hansenne affirme que «libéralisation du commerce international et

17. Bureau imternational du travail, L'action normative de l'OIT à l'heure de la mondialisation, rapport du Directeur général, Conférence internationale du travail, 85 session 1997, Genève, BIT, 1997. 
progrès social doivent aller de pair et qu'il faut des preuves tangibles que les promesses dont celle-ci est porteuse ne soient pas vaines ou illusoires». Pour atteindre ces objectifs le rapport préconise une revitalisation du système des normes internationales du travail, qui comporte:

- un respect universel des droits fondamentaux de l'homme au travail, tels qu'ils sont définis dans les sept conventions fondamentales de ''OIT $^{18}$;

- une «déclaration» solennelle, qui devrait être adoptée en 1998, destinée à compléter la constitution de l'OIT en renforçant les mécanismes de contrôle.

Le rapport propose également la publication régulière de rapports sur les efforts réalisés par chaque pays pour veiller à ce que la croissance économique résultant de la libéralisation des échanges engendre un véritable progrès social et qu'il établisse un système global de labels sociaux garantissant que les biens vendus soient produits dans des conditions humaines. Cette dernière proposition traduit la préoccupation relative à la prolifération de chartes, de codes de conduite et de labels de toutes sortes attestant le respect de certains critères par le fabricant ou le vendeur. Afin d'éviter les risques d'arbitraire, de sélectivité ou de manipulations (ces labels ne sont, par exemple, appliqués qu'aux secteurs d'exportation, les travailleurs produisant pour le marché intérieur se trouvant délaissés), Michel Hansenne est d'avis qu'il serait préférable de décerner un «label social global». Ce label serait attribué aux pays respectant dans chaque secteur les droits et les principes fondamentaux du travail et acceptant de se soumettre à des inspections internationales fiables et juridiquement autonomes.

Les propositions formulées par le Directeur général ont fait l'objet d'interventions parfois passionnées. Pour mener à bien ce débat, il a fallu 11 séances plénières, auxquelles ont pris part 314 orateurs dont 117 ministres. Certains Etats, tels la Chine, Singapour, la Malaisie, le Mexique, l'Iran, la Libye, etc., ont vigoureusement exprimé leur désaccord avec ces propositions, rappelant les arguments de risques protectionnistes déjà évoqués à Singapour. Michel Hansenne, dans son discours final, a reconnu la difficulté du débat, mais selon lui «la discussion a confirmé un large consensus au sujet de l'importance d'un renforcement des droits fondamentaux». Il revient au Conseil d'administration de poursuivre les travaux en vue de l'adoption d'une déclaration solennelle lors de la Conférence de 1998.

Deux mesures ont cependant d'ores et déjà été prises dans ce domaine:

$\checkmark$ une campagne mondiale encourageant les Etats qui ne l'ont pas encore fait à ratifier les sept conventions fondamentales de l'OIT a permis d'obtenir 29 nouvelles ratifications depuis mars 1995 ;

a l'organisation en 1998-99 du Forum social de l'OIT dans le but de promouvoir une meilleure application des normes internationales du travail.

\section{Commission d'application des normes}

Dans le rapport qu'elle a présenté à la Conférence, la Commission d'application des normes a estimé que le non-respect était particulièrement préoccupant en Iran (Convention concernant la discrimination), au Myanmar, au Nigeria et au Swaziland (Convention sur la liberté syndicale et la protection du droit syndical),

18. Liberté syndicale et négociation collective ( $\mathrm{n}^{\mathrm{os}} 87$ et 98 ), travail forcé ( $\mathrm{n}^{\mathrm{os}} 29$ et 105$)$, non-discrimination $\left(\mathrm{n}^{\circ} 100\right.$ et 111), âge minimum ( $\left.\mathrm{n}^{\circ} 138\right)$. 
au Maroc (Convention sur le droit d'organisation et de négociation collective) et au Soudan (Convention sur le travail forcé) ${ }^{19}$.

\section{Programme et budget}

La conférence a adopté un budget de 481 millions de dollars; ce montant représente une diminution de $17 \%$ (98 millions de dollars) par rapport à l'exercice précédent. 5,6\% de cette diminution sont imputables à une réduction des programmes, le reste à l'ajustement du taux de change résultant de l'appréciation du dollar.

Quatre domaines seront prioritaires dans le programme de l'OIT en 1998-99:

๖ la revitalisation de l'action normative de l'Organisation;

- la lutte contre le travail des enfants;

- le suivi du sommet de Copenhague par la promotion d'emplois dans les PME;

- le suivi de la Conférence mondiale des femmes.

\section{Discussion sur les conventions et les recommandations}

La première discussion à laquelle a procédé cette année la conférence concerne le travail en sous-traitance. Cette discussion a ouvert la voie à l'adoption d'une convention et d'une recommandation l'année prochaine, qui visent à garantir une protection adéquate aux travailleurs occupés en sous-traitance.

La Commission de la création d'emplois dans les petites et moyennes entreprises a suggéré l'adoption d'une recommandation pour 1998, document n'ayant pas force obligatoire mais servant de référence à l'échelle internationale. Les PME créent plus de $80 \%$ des emplois dans le monde.

\section{Position suisse}

La Suisse contribue chaque année pour environ 4 millions de francs suisses à l'Organisation internationale du travail et à ses programmes. Lors de la conférence annuelle, la Suisse a approuvé sans réserves le programme et le budget présentés à l'Assemblée générale; elle a également approuvé l'amendement à la Constitution de l'OIT (abrogation des conventions obsolètes). Dans son intervention Jean-Luc Nordmann a soutenu, dans les grandes lignes, les propositions du Directeur général, à l'exception du label social attribué au niveau national, même si la Suisse soutient déjà activement les labels. La délégation gouvernementale suisse a proposé l'organisation d'un forum sur ce thème. JeanLuc Nordmann a également fait part de l'intention de l'OFIAMT d'inviter le Conseil fédéral à soumettre au Parlement la ratification d'importantes conventions de l'OIT sur le droit d'organisation et de négociation collective $\left(n^{\circ} 98\right)$ et sur le travail des enfants $\left(n^{\circ} 138\right)$. Concernant la révision de la Convention $\mathrm{n}^{\circ} 96$ (la nouvelle norme définit des paramètres devant régir les activités des agences privées de placement), la Suisse s'est abstenue.

D'autre part, dans le but de coordonner la position suisse sur la question très controversée de l'introduction de normes sociales dans les échanges commerciaux, un groupe d'experts interdépartemental a été constitué au sein de l'administration fédérale (OFAEE, OFIAMT, DFAE).

19. Pour de plus amples détails: OIT, Communiqué de presse, BIT/97/18, 19 juin 1997, «Violation des droits des travailleurs - Commission d'application des normes». 
Les organisations de travailleurs de Suisse ont salué ${ }^{20}$ l'ouverture de discussions sur la question du travail en sous-traitance et sur les agences de placement. Elles ont également approuvé l'idée, formulée par le directeur du BIT, d'établir un mécanisme de suivi pour rendre compte de l'état du progrès social accompli dans les Etats membres de l'OIT. Enfin, elles ont pris connaissance avec satisfaction de l'intention de l'OFIAMT de faire avancer la question de l'éventuelle ratification des deux dernières conventions fondamentales que la Suisse n'a pas encore ratifiées.

\section{$\square$ Travail des enfants - Conférences d'Amsterdam et d'Oslo}

L'OIT a poursuivi pendant l'année 1997 sa campagne de sensibilisation au travail des enfants, lancée lors de la conférence annuelle en juin 1995, qui doit aboutir à l'adoption d'une nouvelle convention internationale sur les formes les plus intolérables de travail des enfants en 1999. Des réunions internationales ont réuni des représentants de l'OIT, de gouvernements et d'organisations non gouvernementales afin d'alerter l'opinion publique sur la situation de 250 millions d'enfants travailleurs, mais aussi de préparer le terrain pour de nouveaux instruments juridiques. La conférence d'Amsterdam (février 1997) ${ }^{21}$ devait susciter un élan politique nécessaire pour en finir avec l'esclavage, la servitude pour dettes et autres pratiques similaires. La Suisse était représentée par une délégation emmenée par Jean-Luc Nordmann, directeur de l'OFIAMT. En octobre 1997, Oslo $^{22}$ a également accueilli une conférence internationale visant à définir les possibilités d'amélioration de la situation des enfants par le biais de la coopération au développement. Jean-François Giovannini, directeur suppléant de la DDC, a conduit la délégation helvétique. A l'issue de la conférence, les représentants des 41 pays présents ont adopté un plan d'action dont le principal objectif est l'élimination effective et progressive du travail des enfants en donnant une priorité urgente et immédiate à l'abolition des formes les plus intolérables du travail des enfants (esclavage, travail forcé, servitude pour dette, commerce sexuel). Par ailleurs, le programme préconise la révision des programmes existants de coopération pour le développement, aussi bien bilatéraux que multilatéraux, afin d'évaluer leur impact sur le travail des enfants. De plus, il recommande aux pays de prendre une part active à l'élaboration et à l'adoption d'une nouvelle convention du BIT sur les formes les plus extrêmes de travail des enfants, qui doit être discutée lors de la prochaine conférence internationale du travail qui se tiendra à Genève en 1998. A la suite de la conférence d'Oslo, la Suisse a annoncé qu'elle va renforcer ses moyens de lutte contre l'exploitation des enfants et mettre au point une nouvelle stratégie d'aide au développement social.

En mai 1997, des représentants de 20 pays latino-américains se sont également réunis à Cartagena $(\text { Colombie })^{23}$, préoccupés par l'augmentation du travail des enfants dans la région sud-américaine. Un rapport publié par l'OIT, Combattre les formes les plus intolérables du travail des enfants, un défi universel ${ }^{24}$, sert de base pour la nouvelle convention et les travaux des conférences.

20. USS, FSE, CSC, Communiqué de presse, Berne, mai 1997, «Convertir en progrès social les dividendes de la globalisation». 21. OIT, Communiqué de presse, BIT/97/3, 25 février 1997, "Conférence d'Amsterdam».

22. OIT, Communiqués de presse relatifs à la conférence d’Oslo, BIT/97/22, 14 octobre 1997, BIT/97/26, 29 octobre 1997, BIT/97/27, 30 octobre 1997.

23. OIT, Communiqué de presse, BIT/97/11, 7 mai 1997, «Quinze millions d'enfants travaillent en Amérique latine».

24. Bureau international du travail, Combattre les formes les plus intolérables du travail des enfants, un défi universel, Genève, BIT, janvier 1997. 


\section{Rapport sur le travail dans le monde 1997-1998}

En novembre 1997, le BIT a publié une nouvelle édition de son rapport Le travail dans le monde, avec pour sous-titre Relations professionnelles, démocratie et cohésion sociale. Il est consacré aux syndicats et aux organisations d'employeurs dans un contexte de mutations économiques et sociales. Premier constat, les effectifs syndicaux à travers le monde ont diminué d'environ $10 \%$ de 1985 à 1995. Seulement 12,6\% des 1,3 milliard de travailleurs, soit 164 millions de personnes, sont syndiqués. Ce déclin est en grande partie imputable à la réduction des emplois dans le secteur public et manufacturier. La mobilisation croissante offre une plus grande autonomie aux entreprises. Les syndicats ont en outre négligé de s'adresser aux jeunes ou aux femmes, ainsi qu'aux petites et moyennes entreprises. En Suisse, selon les statistiques du BIT, la baisse a été de $21,7 \%$ avec en 1997 un taux de 22,5\% de travailleurs syndiqués (soit 740'000 personnes). Présentant les résultats de cette étude, Michel Hansenne a interprété ces résultats comme étant le signe d'un changement radical des relations professionnelles, mais non une perte de pouvoir des syndicats à l'échelle mondiale. Le rapport relève que dans la plupart des pays l'attitude des syndicats a évolué vers moins d'antagonisme et plus de coopération; ceux-ci se sont montrés capables de s'imposer en période de crise. D'autre part, les syndicats ont beaucoup développé leur action à l'échelle internationale, allant jusqu'à obliger des entreprises multinationales (comme Nike, Gap et d'autres) à adopter des «codes de conduite» applicables aux travailleurs et aux sous-traitants auxquels elles font appel dans le monde entier.

\section{$\square$ Contributions des organisations non gouvernementales}

A l'initiative des organisations non gouvernementales suisses, le Forum «Commerce international et normes sociales» regroupe des représentants d'ONG, des syndicats et de l'administration fédérale. Le forum fonctionne avec des groupes de travail thématiques et publie une newsletter faisant un inventaire régulier sur l'état de la réflexion au sein des organisations internationales, de l'administration fédérale et de la société civile ${ }^{25}$.

En février 1997, la Déclaration de Berne et Terre des hommes-Suisse ont lancé une campagne d'information, Let's go fair, dénonçant les conditions de travail dans les usines de chaussures de sport d'Asie du Sud-Est. Les consommateurs étaient invités à renvoyer aux fabricants et distributeurs de chaussures une carte postale leur demandant de signer des codes de conduite reprenant les conventions fondamentales de l'OIT et d'accepter des contrôles indépendants pour en vérifier la mise en œuvre. Plus de 40’000 cartes postales ont été envoyées aux différentes firmes, qui ont répondu à chacune d'entre elles. Fortes de la pression des consommateurs, il s'agit à présent pour les organisations d'entrer en dialogue avec ces entreprises afin de les inciter à adopter un code de conduite et un système de contrôle indépendant et transparent ${ }^{26}$. 


\section{SOURCES}

Bureau international du travail, Compte-rendu provisoire, « $85^{\circ}$ session de la Conférence internationale du travail», Genève, BIT, juin 1997.

Bureau international du travail, Communiqués de presse publiés en 1997.

Bureau international du travail, Le travail dans le monde 1997-98 - Relations professionnelles, démocratie et cohésion sociale, Genève, BIT, novembre 1997.

Bureau international du travail, L'emploi dans le monde 1996-97 - Les politiques nationales à l'heure de la mondialisation, Genève, BIT, octobre 1996.

Bureau international du travail, L'action normative de l'OIT à l'heure de la mondialisation, rapport du Directeur général, Conférence internationale du travail, 85 session 1997, Genève, BIT, 1997.

Travail - Le magazine de l'OIT, $\mathrm{n}^{\circ} 18$ - décembre 1996, n 19 - mars 1997, n²0 - juin 1997.

Agefi, 23 avril 1997 «Interview de Michel Hansenne», 4 novembre 1997 «Les relations du travail s'individualisent toujours plus".

Journal de Genève, 26 février 1997 (conférence d'Amsterdam), 23 avril 1997 «Pour en finir avec la clause sociale, le BIT table sur l'opinion publique», 13 juin 1997 «Les économies émergentes rejettent l'idée d'unlabel social global», 19 juin 1997 «La conférence du travail s'achève en adoptant un profil bas», 28 octobre 1997 «La conférence d'Oslo déclare hors la loi le travail des enfants», $1^{\text {er }}$ novembre 1997 «La Suisse veut renforcer la lutte contre l'exploitation des enfants», 4 novembre 1997 «En crise, les syndicats coopèrent pour maintenir leur influence».

La Liberté, 23 avril 1997 «Le BIT pour des règles minimales».

Neue Zürcher Zeitung, 16. Januar 1997 «ILO-Reaktion auf die Welthandelskonferenz», 23. April 1997 «Ein Konzept für eine ILO-Teilreform», 4. Juni 1997 «Die ILO auf dem Weg in die Zukunft», 20. Juni 1997 «Modernisierungspläne der ILO".

\section{SITE INTERNET}

http://www. unicc. org/ilo

L'OIT est présente sur le web depuis le mois de mars 1996. Au départ ce site contenait quelques documents de presse, des publications et des fiches de données sur l'histoire et les aspects essentiels du mandat de l'OIT. Aujourd'hui le site compte 1500 documents (dont des bases de données sur les normes internationales de travail, sur les lois nationales de travail, documentation des conseils d'administration, etc.). 